\title{
Técnicas de sensoriamento remoto para delimitação de áreas queimadas no Tocantins através do processamento digital de imagens Landsat 8-TM
}

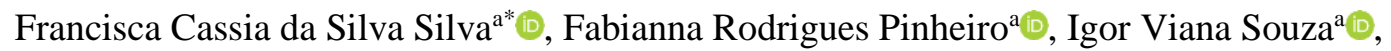 \\ Victor Braga Rodrigues Duarte ${ }^{\mathrm{a}} \odot$, Marcos Vinícius Cardoso da Silva ${ }^{\mathfrak{\oplus}}$, Augustus Portella ${ }^{\mathfrak{a}}$, \\ Marcos Giongo ${ }^{a}$ (C) \\ ${ }^{\text {a } U n i v e r s i d a d e ~ F e d e r a l ~ d o ~ T o c a n t i n s, ~ B r a s i l ~}$ \\ *Autor correspondente (fcassia.silv@gmail.com)
}

\section{N F O}

\section{Keyworks}

detection

brazilian savannah

fire use

\section{Palavras-chave}

detecção

savana brasileira

uso do fogo

\begin{abstract}
A B S T R A C T
Remote sensing techniques for delimitation of burned areas in Tocantins through Landsat 8-TM digital image processing

Methods of detection of burned areas are fundamental for a historical survey, as well as for the sizing of the effects produced by fire on the environment. In this sense, the objective of this work was to evaluate the procedure and methodology for the delimitation of burned areas by processing Landsat 8-TM images, during the period of greatest occurrence of hot flashes in three areas, which are located in the Eastern, Southern and Northern regions of the state of. Tocantins. To obtain the burned areas, image classification procedures were performed aiming at the class of interest (burned area). Initially, pre-processing was performed on the images: radiometric, atmospheric correction and projection system correction. After this step, the images were processed to obtain the Normalized Difference Vegetation Index (NDVI) in images before and after fire, called $\operatorname{Im} 1$ and $\operatorname{Im} 2$. After obtaining the NDVI, the images were compared pixel by pixel by subtracting the NDVI values, generating a difference image. Supervised rating shows better performance than unsupervised. The highest occurrences of burnt areas were in scenes 221/67 and 222/68 which is related to the climate, vegetation, occupation and land use of the regions covered by these scenes.
\end{abstract}

\section{R E S U M O}

Métodos de detecção de áreas queimadas são fundamentais para que se possa realizar um levantamento histórico, bem como para o dimensionamento dos efeitos produzidos pelo fogo sobre o ambiente. Nesse sentindo o objetivo desse trabalho foi avaliar procedimento e metodologia para a delimitação de áreas queimadas através do processamento de imagens Landsat 8-TM, no período de maior ocorrência de focos de calor em três áreas, sendo estas localizadas nas regiões Leste, Sul e Norte do estado do Tocantins. Para a obtenção das áreas queimadas foram realizados procedimentos de classificação das imagens objetivando a classe de interesse (área queimada). Inicialmente foi realizado pré-processamento nas imagens sendo: correção radiométrica, atmosférica e correção do sistema de projeção. Após esta etapa, foi realizado o processamento das imagens para a obtenção do NDVI (Normalized Difference Vegetation Index) em imagens antes e depois do fogo, denominadas de Im1 e Im2. Após a obtenção do NDVI, as imagens foram comparadas pixel a pixel por subtração dos valores de NDVI, gerando uma imagem diferença. A classificação supervisionada apresentou melhor desempenho que a não supervisionada. As maiores ocorrências de áreas queimadas foram nas cenas 221/67 e 222/68 que está relacionada com o clima, vegetação, ocupação e uso do solo das regiões abrangidas por estas cenas. 


\section{INTRODUÇÃO}

O cerrado vem sofrendo distúrbios devido a ação humana podendo ter relação com o fato de que embora o fogo ocorra de forma natural neste bioma, seu uso é comumente associado a agricultura. Esta ação, em geral, está voltada para a ampliação de áreas para renovação de pastagens, seja para criação de gados ou para rotação de culturas, podendo ocasionar prejuízos aos recursos naturais. De acordo com Goulart et al. (2011), as transformações ocorridas no cerrado se devem ao uso intensivo do fogo, e ainda, segundo França et al. (2007) fatores como clima seco, predominância de gramíneas e materiais inflamáveis podendo ocasionar o incidente de focos de incêndios.

Nesse sentindo o uso de dados oriundos do sensoriamento remoto se tornam relevantes, sendo assim fonte de informações sobre os vários fenômenos que ocorrem na superfície terrestre. Estes dados permitem desenvolver estudos das características espectrais do uso da terra, bem como da cobertura do solo, movimentos de massa, rochas, solos, expansão de áreas agrícolas e florestas, monitoramento de inundações e secas, análise temporal da paisagem e detecção e estimativa de áreas queimadas.

No entanto, a falta de informações adequadas da localização dos incêndios florestais e sua extensão em termos de área queimada prejudica a estimativa do impacto do fogo sobre o ambiente. Portanto, métodos de detecção de áreas queimadas são fundamentais para que se possa realizar um levantamento histórico, bem como para o dimensionamento dos efeitos produzidos pelo fogo sobre o ambiente. Neste ponto, a delimitação das áreas queimadas a partir de dados do sensoriamento remoto vem se desenvolvendo notavelmente nos últimos anos, decorrente de um grande interesse em se obter avalição das áreas afetadas pelos incêndios florestais (Ahern et al., 2001).

A utilização de imagens de média resolução espacial na quantificação de áreas queimadas apresenta desvantagem relacionada a presença de nuvens e a baixa frequência temporal. Imagens com alta frequência temporal geralmente possuem baixa resolução espacial e recomenda-se o uso das imagens de média/alta resolução para validação dos dados (Boschetti et al., 2007).

O estado do Tocantins é considerado, entre os estados brasileiros, um dos mais impactados em incêndios florestais, possuindo inúmeras áreas de difícil acesso que frequentemente apresentam ocorrências do fogo (acima de 225 mil focos de calor no período de 1998 a 2016, segundo dados do INPE) e o uso de dados de sensores orbitais pode ser fonte de informação importante para o entendimento da dinâmica destes eventos e subsidiar as ações de prevenção e combate de incêndios florestais, bem como a definição de políticas públicas regionais. Levando em consideração que o clima seco nos meses de junho a setembro ocasiona maior risco de incêndios (INPE, 2016; Pivello, 2011).

Neste sentido, se faz necessário a realização do procedimento e metodologia para a delimitação de áreas queimadas através do processamento digital de imagens Landsat/ TM, a fim de automatizar o processo de análises das imagens e consequentemente melhorar os resultados finais. Desta forma o objetivo desse trabalho foi avaliar procedimento e metodologia para a delimitação de áreas queimadas através do processamento de imagens Landsat, no período de maior ocorrência de focos de calor em três áreas distintas no estado do Tocantins.

\section{MATERIAL E MÉTODOS}

$\mathrm{O}$ estado do Tocantins localiza-se na região Norte do país, ocupando uma área com 227.620 $\mathrm{km}^{2}$, entre os paralelos $5^{\circ} 10^{\prime} 06^{\prime \prime}$ e $13^{\circ} 27^{\prime} 59^{\prime \prime}$ de latitude $S$, e entre os meridianos $45^{\circ} 44^{\prime} 46^{\prime \prime}$ e $50^{\circ}$ $44^{\prime} 33^{\prime \prime}$ de longitude $\mathrm{O}$, com temperatura média $32^{\circ}$ $\mathrm{C}$ na seca e $26^{\circ} \mathrm{C}$ no período de chuvoso (SEPLAN, 2015). Para realização das avaliações de metodologia de processamento de imagens, visando a delimitação de cicatrizes de incêndios florestais, foi utilizado 3 cenas (Figura 01).

- Cena 1: 221/67, localizada ao Leste, abrangendo 7 municípios;

- Cena 2: 222/68, localizada ao Sul, abrangendo 13 municípios;

- Cena 3: 223/65, localizada ao Norte, abrangendo 23 municípios.

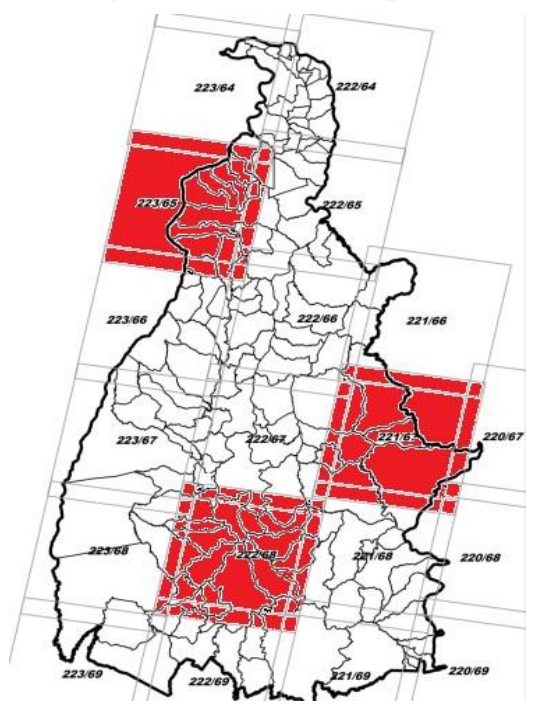

Figura 01 - Localização das cenas. 
Em cada cena foram utilizadas imagens pré e pós fogo que estão descritas na tabela 01 .

Tabela 01 - Cenas utilizadas para a realização do estudo.

\begin{tabular}{ccc}
\hline \multirow{2}{*}{ Cena } & \multicolumn{2}{c}{ Datas } \\
\cline { 2 - 3 } & Pré- fogo & Pós-fogo \\
\hline $221 / 67$ & $03 / 06 / 2016$ & $05 / 08$ e 07/09/2016 \\
$222 / 68$ & $25 / 06 / 2016$ & $29 / 08$ e $14 / 06 / 2016$ \\
$223 / 65$ & $03 / 07 / 2016$ & $20 / 08$ e $21 / 09 / 2016$ \\
\hline
\end{tabular}

Para a obtenção das áreas queimadas foram realizados procedimentos de classificação das imagens objetivando a classe de interesse (área queimada). Inicialmente foi realizado pré-processamento nas imagens sendo: correção radiométrica e atmosférica e correção do sistema de projeção. Para composição das cenas e para delimitação visual da área queimada, foram utilizadas as composições RGB: 653 e 754 e que apresentam, respectivamente, as seguintes faixas do espectro: Banda 3 - Visível Verde $(0,53-0,59 \mu \mathrm{m})$, Banda 4 - Visível Vermelho $(0,64-0,67 \mu \mathrm{m})$, Banda 5 - Infravermelho Próximo $(0,85-0,88 \mu \mathrm{m})$, Banda 6 - Infravermelho Médio (1,57 - 1,65 $\mu \mathrm{m})$ e a Banda 7 - Infravermelho Médio $(2,11-2,29 \mu \mathrm{m})$.

Após esta etapa, foi realizado o processamento das imagens para a obtenção do NDVI (Normalized Difference Vegetation Index) em imagens antes e depois do fogo, denominadas de $\operatorname{Im} 1$ e Im2, respectivamente. O índice (NDVI) foi obtido através da seguinte fórmula:

$$
N D V I=\frac{(N I R-V I S)}{(N I R+V I S)}
$$

NDVI = Índice de normalização da diferença da vegetação.

NIR $=$ Refletância no infravermelho próximo, banda 4 do sensor TM $0,76-0,90 \mu \mathrm{m}$.

VIS $=$ Refletância no infravermelho, banda 3 do sensor TM 0,63 $0,69 \mu \mathrm{m}$.

Após a obtenção do NDVI, as imagens foram comparadas pixel a pixel por subtração dos valores de NDVI, gerando uma imagem diferença. Nesta imagem, os valores próximos de zero indicam áreas onde não ocorreram mudanças na cobertura da terra. Valores positivos indicam áreas onde houve diminuição da cobertura vegetal e valores negativos indicam áreas onde houve um crescimento expressivo da cobertura vegetal (Carvalho, 2008).

A partir da imagem diferença foi realizada a classificação não supervisionada em que consistiu na utilização de algoritmos para reconhecimento das classes presentes na imagem (Xu e Wunsch, 2005) , seguido do pós-processamento de classificação como análise de maioria-minoria e a combinação de classes semelhantes para correção de possíveis erros tendo como resultado final a delimitação dos polígonos de áreas queimadas. A metodologia desse trabalho, para a obtenção dos polígonos das cicatrizes de áreas queimadas, pode ser verificada na figura 02 que representa o diagrama de fluxo do processamento:

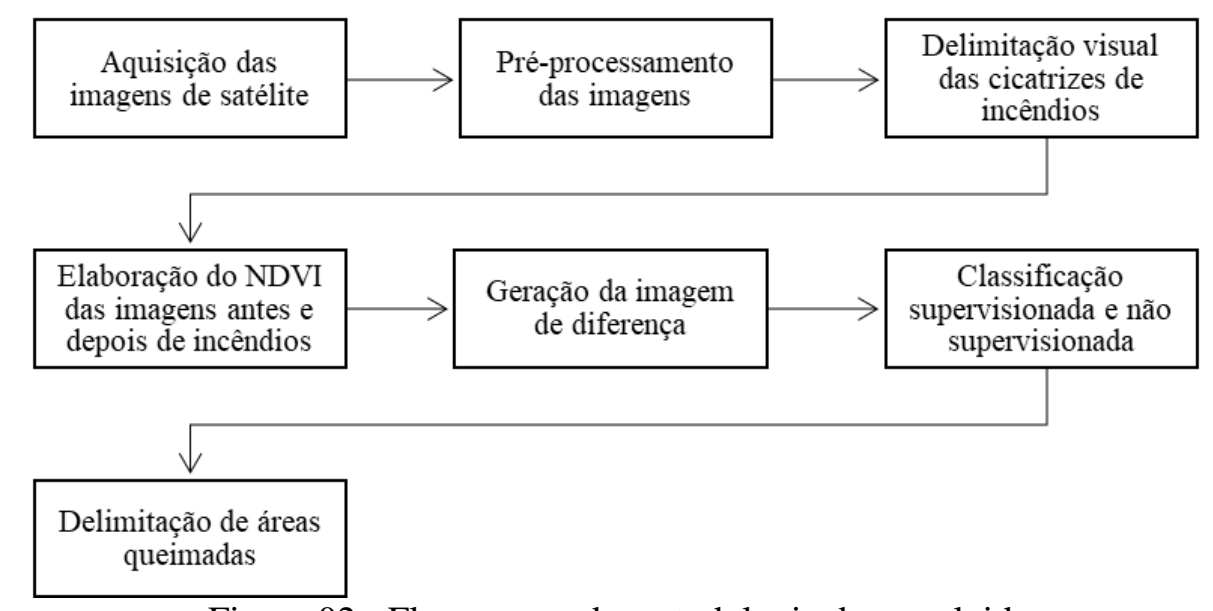

Figura 02 - Fluxograma da metodologia desenvolvida.

A avaliação da eficiência do método de classificação não supervisionada foi feita por meio da matriz de erro com a área queimada delimitada visualmente. $\mathrm{O}$ emprego da matriz de erro torna-se uma das técnicas mais utilizadas na realização de avaliação de acurácia da classificação de dados de sensoriamento remoto e que, também, pode ser conhecida como matriz de confusão (Congalton, 1991). Esta é uma matriz quadrada de números definidos 
em linhas e colunas que expressam o número de unidades da amostra (pixels, grupos de pixels ou polígonos) atribuído a uma categoria particular relativo à categoria atual.

Assim, foram estimados erros de inclusão (erros de comissão) e o de exclusão (erros de omissão) presentes na classificação, em que um erro de omissão ocorre ao incluir um objeto na classe à qual ele não pertence e o de omissão quando um objeto é excluído da classe a que pertence.

\section{RESULTADOS E DISCUSSÃO}

A classificação manual (acerto < 42,76\%) apresentou melhor resultado quando comparada a classificação não supervisionada (Tabela 2), diversos fatores podem influenciar nesses resultados, como solo, clima e vegetação. Com relação a área queimada delimitada visualmente, as maiores ocorrências foram nas cenas 221/67 e 222/68 que apresentaram mais de 119 mil hectares de área queimada. Tal resultado possui relação com o clima, vegetação, ocupação e uso do solo destes dois locais do estado do Tocantins (região leste 221/67; região sul - 222/68). A cena 221/67 apresentou maior área queimada tanto na classificação supervisionada como não supervisionada isso deve ao fato dessa região apresentar maior período de estiagem , tendo em vista que a escassez de chuva pode influenciar em um maior número de queimadas (NATURATINS, 2003).

Tabela 02 - Área queimada delimitada manualmente e por meio de classificação não supervisionada (NDVI), em hectares e seus erros de omissão e comissão e percentagem de acerto.

\begin{tabular}{cccccc}
\hline \multirow{2}{*}{ CENA } & \multicolumn{2}{c}{ Área Queimada (ha) } & \multicolumn{2}{c}{ Erros $(\%)$} & \multirow{2}{*}{ Acerto (\%) } \\
\cline { 2 - 5 } & Manual & NDVI & Omissão & Comissão & \\
\hline $221 / 67$ & $120.539,35$ & $165.140,41$ & 65,88 & 102,88 & 34,12 \\
$222 / 68$ & $119.281,11$ & $121.796,51$ & 57,24 & 59,21 & 42,76 \\
$223 / 65$ & $7.988,98$ & $34.846,24$ & 95,41 & 99,02 & 4,59 \\
\hline
\end{tabular}

Segundo Eva e Lambin (2000) e Ometto et al. (2014), em áreas de transição há um aumento nas áreas queimadas, que está intimamente ligado com as mudanças nas últimas décadas em uso e cobertura do solo. Por exemplo, de acordo com Silva (2007), o estado do Tocantins apresenta faixas de transições entre o bioma Amazônia e o Cerrado, com predomínio deste último e que segundo dados publicados por Fanin e Van der Werf (2015), a região de savana (Cerrado), que inclui
Maranhão e Tocantins, apresentou maior concentração de áreas queimadas de 2002 a 2012, com base no produto MCD64A1.

$\mathrm{Na}$ figura 03 é possível verificar que a cena 223/65 apresentou menor área queimada na delimitação manual, podendo ser explicado pelos diferentes aspectos geológicos e geomofológicas entre os rios Tocantins e Araguaia, conferindo assim uma vegetação bastante variada na área, apresentando Floresta Ombrófila Aberta e Densa, Floresta Estacional Semidecidual (SEPLAN, 2012).
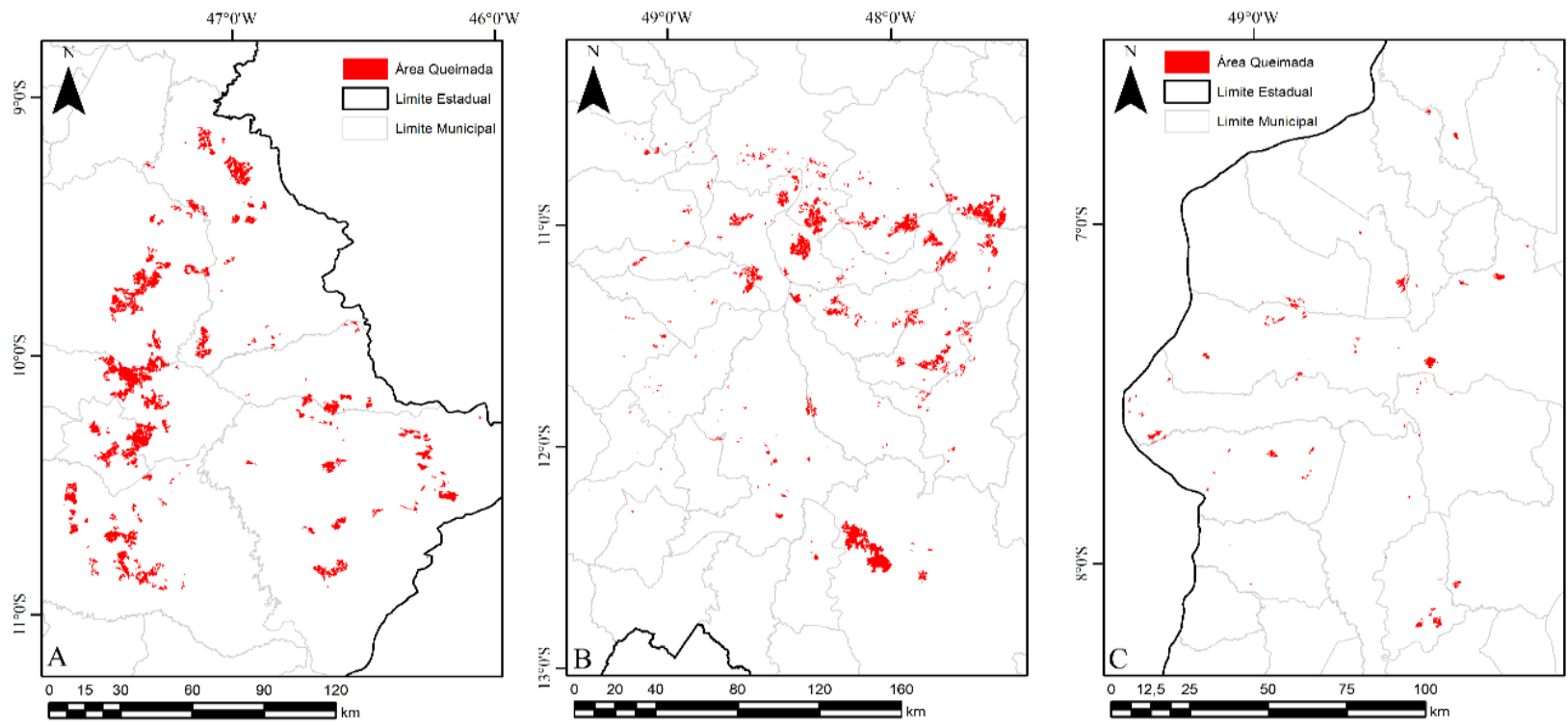

Figura 03 - Área queimada delimitada manualmente em três áreas distintas do estado do Tocantins. 
No tocante aos locais de maior ocorrência de área queimada neste estudo, Libonati et al. (2015) relatam que a presença de estação seca dos meses de maio a setembro e que anos com menores precipitações foram os que apresentaram maiores áreas queimadas, como também observado por Araújo e Ferreira (2015), com relação aos meses do ano. No entanto, Libonati et al. (2015) destacam que além do clima, o fator humano exerce influência no regime do fogo.

Phulpin et al. (2002) relatam que as imagens de satélites têm sido utilizadas para monitorar incêndios, estudos climáticos e avaliação dos impactos das queimadas. Os dados, coletados através dessas imagens, são de grande importância pois servem de alerta para as autoridades durante o período em que as queimadas ocorrem. De acordo com Hardesty et al. (2005), as influências humanas no regime do fogo ocorrem de forma direta e indireta em que o aumento direto no número de ignições está associado principalmente com limpeza de áreas para pastagem, preparação de terras para agricultura e atração animal para a caça, situações em que o fogo pode sair do controle e gerar grandes incêndios florestais.

\section{CONCLUSÕES}

A classificação supervisionada apresentou melhor desempenho que a não supervisionada para a identificação de áreas queimadas nas três regiões distintas do Estado do Tocantins, necessitando de ajustes para o local de estudo. As maiores ocorrências de áreas queimadas foram nas cenas 221/67 e 222/68 que está relacionada com o clima, vegetação, ocupação e uso do solo das regiões abrangidas por estas cenas.

\section{REFERÊNCIAS BIBLIOGRÁFICAS}

Ahern FJ, Goldammer JG, Justice CO. Global and regional vegetation fire monitoring from space: planning a coordinated international effort. Holanda: Kugler Publications, 303p. 2001.

Araújo FM, Ferreira LG. Satellite-based automated burned area detection: A performance assessment of the MODIS MCD45A1 in the Brazilian savanna. International Journal of Applied Earth Observation and Geoinformation, v. 36, p. 94-102, 2015.

https://doi.org/10.1016/j.jag.2014.10.009.

Boschetti L, Eva HD, Brivio PA, Grégoire JM. Lessons to belearned from the comparison of three satellite-derived biomas burnings pruducts. In: Setzer, A.; Morelli, F.; Lombardi, R. R; (Org.). O sensor MODIS e suas aplicações no Brasil. São José dos Campos: Editora Parêntese, 2007. cap. 28, p. 403-417.
Brasil. Ministério do Meio Ambiente. Mapeamento do uso e cobertura do cerrado: Projeto TerraClass Cerrado 2013. Brasília: MMA, 2015.

Carvalho LMT, Scolforo JRS. Inventário Florestal de Minas Gerais: Monitoramento da Flora Nativa. 2005-2007. Lavras: Editora UFLA, 318p. 2008.

Congalton RG. A review of assessing the accuracy of classifications of remotely sensed data. Remote Sensing of Environment, v.49, n.12, p.1671-1678, 1991.

Eva H, Lambin EF. Fires and land-cover change in the tropics: a remote sensing analysis at the landscape scale. Journal of Biogeography, v. 27, n. 3, p. 765-776, 2000. https:// doi:10.1046/j.1365-2699.2000.00441.x.

Fanin T, Van der Werf GR. Relationships between burned area, forest cover loss, and land cover change in the Brazilian Amazon based on satellite data. Biogeosciences, v.12, p.6033-6043, 2015. https:// doi:10.5194/bgd-12-8235-2015.

França H, Neto MBR, Setzer A. O fogo no Parque Nacional das Emas. Brasília: MMA, 140p., 2007.

Goulart AA, Morelli F, Setzer A. Focos de queimadas e incêndios detectados por satélites nas unidades de conservação do Cerrado no Mato Grosso em 2010. In: Simpósio Brasileiro de Geografia Física Aplicada, 14, 2011, Dourados. Anais. Mato Grosso do Sul: UFGD.

Hardesty J, Myers R, Fulks W. Fire, ecosystems and people: a preliminary assessment of fire as a global conservation issue. Fire Manage, v.22, p.78-87, 2005.

Instituto Nacional de Pesquisas Espaciais (INPE). Portal Programa Queimadas. 2016. Disponível em: Acesso 31 nov. 2019.

Libonati R, Camara CC, Setzer AW, Morelli F, Melchiori AE. An algorithm for burned area detection in the Brazilian Cerrado using $4 \mu \mathrm{m}$ MODIS imagery. Remote sensing, v.7, p.15782-15803, 2015. https://doi:10.3390/rs71115782.

NATURATINS - Instituto Natureza do Tocantins. Plano de Manejo do Parque Estadual do Jalapão-PEJ. Palmas: Naturatins, 2003. 204 p.

Ometto JP, Aguiar AP, Assis T, Soler L, Valle P, Tejada G, Lapola DM, Meir P. Amazon forest biomass density maps: tackling the uncertainty in carbon emission estimates. Climatic Change, v.124, n.3, p.545-560, 2014. http://dx.doi.org/10.1007/s10584-014-1058-7.

Phulpin T, Lavenu F, Bellan MF, Mougenot B, Blasco F. USING SPOT-4 HRVIR and VEGETATION sensors to assess impact of tropical forest fires in Roraima, Brazil. International Journal of Remote Sensing, v.23, n.10, p.19431966, 2002.

https://doi.org/10.1080/01431160110076135.

Pivello V. The use of fire in the Cerrado and Amazonian Rainforests of Brazil: past and present. Fire Ecology, v.7, n.1, p.25-39, 2011. https://doi: 10.4996/fireecology.0701024.

Secretaria do Planejamento Superintendência do Planejamento e Gestão Central de Políticas Públicas (SEPLAN). Diretoria de Zoneamento Ecológico-Econômico (DEZ). 
Base de dados geográficos do Tocantins, Palmas, SE-

PLAN/DEZ, 2015.

SEPLAN. Secretária de Planejamento do Estado do Tocantins. Atlas do Tocantins: subsídios ao planejamento à gestão territorial. $6^{\circ}$ edição. Palmas. 2012. 80p.

Silva LAGC. Biomas presentes no estado de Tocantins. Brasília: Consultoria Legislativa, 2007. 10 p. (Nota técnica). Acesso em: 20 out. 2019. Disponível em: <https://goo.gl/ENNyjd>.

$\mathrm{Xu} \mathrm{R}$, Wunsch ID. Survey of clustering algorithms. IEEE Transactions on Neural Networks. v.16, n.3, p.645-678, 2005. 